\title{
Design and develop Quality of Service Framework using Fog Computing for Smart City Applications
}

\author{
Prabhdeep Singh, Rajbir Kaur
}

\begin{abstract}
In today's world, Internet of Things (IoT) is has become the most promising and life-changing technology. In the past few years, IoT has become most productive in the area of healthcare, to improve the quality of care to the patients. This paper aims to reduce the delay, energy consumption of cloud data-centers and minimized the power consumption IoT devices using fog devices. To solve the problem mentioned above, we proposed the Quality of Service framework using fog computing for smart city applications named FATEH, a three-tier architecture for IoT-based application. Various quality of services parameters are optimized as For minimizing the power consumption of IoT devices, the Routing Protocol for Low power and Lossy network (RPL). The other QoS parameter is computing the performance of the proposed framework which has been evaluated through the iFogsim toolkit and the Cooja simulator. Results show the efficient reduction in the delay as well as energy consumption in the proposed scenario and provide better QoS framework
\end{abstract}

Keywords: Fog computing, Cloud computing, IoT, Healthcare, Cooja.

\section{INTRODUCTION}

In today's era, connected devices combined with cloud computing have become the pioneer for providing information accessible worldwide. The devices across the globe are predicted to be than 5 billion by 2020 . In cloudbased systems, the increasing workload on cloud datacenters leads to introduce network congestion, high delay, and more energy consumption of data-centers [5].

In this paper, Gog assisted Quality of Service framework/architecture is proposed for smart city applications named FATEH. To improve the Quality of services, various parameters are analyzed. The focus of the FATEH is to minimize the delay as well as the energy consumption of cloud data-centers and IoT devices. It has three-layers. The first layer is AgentNode and IoT devices to collect the end-user data for further processing. An agent node is capable of collecting the data from all the IoT devices. RPL protocol is utilized to minimize the energy consumption of such IoT devices. At the second layer, SDN-based SmartFogNode is proposed the which is used for preprocessing of the data received from the AgentNode. It consists of network edge devices for the local processing of data at the extreme edge. The uppermost layer has cloud

Revised Manuscript Received on November 12, 2019.

* Correspondence Author

Prabhdeep Singh, Department of Computer Science and Engineering, Punjbai University Patiala, India. Email: ssingh.prabhdeep@gmail.com

Rajbir Kaur, Department of Electronics and Communication, Punjbai University Patiala, India. Email : rajbir277@yahoo.co.in data-centers, where the final processing of data is completed. The remainder organization of this paper has following sections. Related work is presented in Section II. Further, a secure IoT based Energy-Efficient cloud-fog Architecture is proposed in section III. Section IV has the performance evaluation is presented by simulating the proposed architecture in ifogSim and Contiki Cooja. Section V represents Result \& Discussion . Finally, at last, section VI presents the conclusion of the paper.

\section{RELATEd WORK}

It is observed that all the above ongoing issues were resolved by using the integration of several technologies and communications solutions. Almost all of the research focused on storage, cost reduction, and power consumption of cloud data-centers as well as IoT devices. There is a lot of work that is still required to address such challenges using SDN based fog nodes for processing IoT devices and fog/cloud datacenters. Therefore, a framework is proposed which efficiently achieve all the above said essential requirements. Gubbi et al. [7] implemented the IoT in the Cloud-centric environment worldwide. They discussed various technologies that drive IoT research in the current future. The interaction between private cloud and the public cloud was implemented using Aneka. Premsankar et al. [13] advocated edge computing for promising IoT applications to boost interactive applications. They provided a survey on present edge computing architectures and then described the benefits of edge computing in different scenarios. In their experimental evaluation, results show the importance of edge computing for reducing latency of applications. Ning et al.[11] introduced the need for training in colleges by using the rapidly developing technologies like fog, cloud, and IoT. They proposed two models for future IoT for (1) establishing and classifying the complicated IoT technologies and (2) building future IoT system architecture

for a new educational system.

Bonomi et al. [2] examined the IoT disruptions in cloud computing and proposed a hierarchical distributed architecture that extended it to the term called Fog Computing. Also, they highlighted new dimensions to add Big Data and Analytics to the IoT. Jalali et al. [8] proposed an energy-efficient model that is based on both flow and time. In their experiment, the energy consumed by the fog based nodes and cloud-based data centers is compared. Vaquero and Merino [14] presented fog

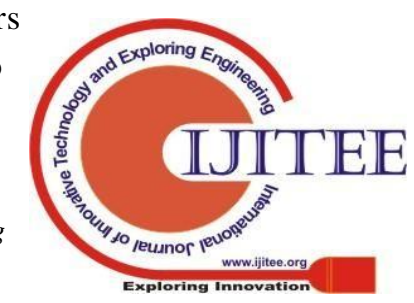


computing, where routers themselves may become the virtualization infrastructure at the edge of the network. Further, they provided a comprehensive definition of the fog in the context of cloud, sensor networks, peer-to-peer networks, and network virtualization functions economic Yang et al. [16] proposed architecture for data streaming by examining the most popular properties of several typical applications. Authors also analyzed the design space of data streaming with the consideration of essential features where both new design difficulties and the issues that arise from leveraging existing techniques are investigated.

Peng et al.[12] proposed the economic spectral efficiency (ESE) to reduce the wired/wireless front haul cost of the cloud paradigm. They formulate the power consumption in data transmission. To deal with this non-convexity, an algorithm is proposed based on both outer and inner loops. $\mathrm{Li}$ et al. [9] investigated the role of object virtualization concept for creating tailored IoT based educational applications for end-users. They studied network function virtualization for performing the flexibility of network service provisioning. Further, they proposed a virtual Fog framework that can be used for verification and conducting quantitative analysis. It is demonstrated that their framework proves efficiency in minimizing latency, operating expense and maximizing multitenancy IoT based applications.

\section{PRoposed Model}

The architecture for smart city application of the proposed framework FATEH is depicted in Figure 1which is focused on improving the QoS parameters. It uses a three-layered architecture. The first layer consists of AgentNode and IoT devices that collect the data and submit to the second layer based on the action required. These IoT devices sense the environment and send observed values to the fog layer via AgentNode for further processing. The next layer, a fog manager, is proposed that efficiently process the request on SmartFogNode at the fog layer. The third layer is used for processing and storing the less- time-sensitive data as wellas processed data coming from the fog manager. All three layers are explained in detail.

\section{A. Physical Layer}

This layer consists of different kinds of IoT devices used in various applications. An AgentNode is placed in this layer, which is responsible for collecting the data from the various geographicallydistributed IoT devices. For the same, various wireless communication mediums can be used to transfer the forwarded to the fog manager located at the next layer.

\section{B. Fog Layer}

This layer is monitored by the proposed fog manager that analyzes and classifies the request based on time sensitivity. The data is categorized by the fog manager as real-time sensitive and less-time sensitive data. The real time-sensitive data is processed by the fog nodes that are closest to the devices that are generating the data. The second type of data that can tolerate minutes of processing is called less-time data such as 3G/4G/CDMA/GPRS. The data is collected and

sensitive data, sent to the cloud data-centers for processing and further analysis. The fog manager is the core component that manages resources of the fog layer in such a way that Quality of Service (QoS) And optimize resource utilization is optimized. The flow chart for allocating the coming request to the SmartFognode at the fog layer is shown in Figure 2.

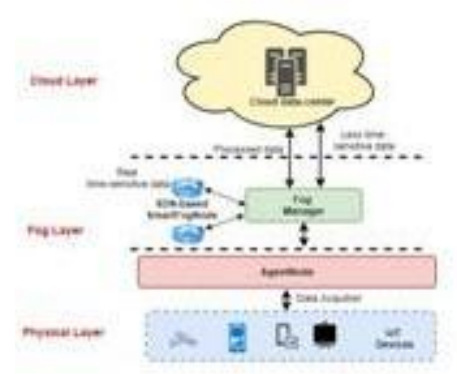

Figure 1: A Fog Assisted IoT based Energy-efficient architecture.

The real time-sensitive applications that need immediate processing are processed by the fog layer. This layer is responsible for the preliminary processing to be completed and appropriate decision making. The processing and storage requirements of the real-time applications are fulfilled by aggregating the computing capabilities of network elements residing at the fog layer. The processed data or results are stored in the repository of the cloud datacenters. On the other hand, the less-time sensitive data is handled at the cloud data-center.

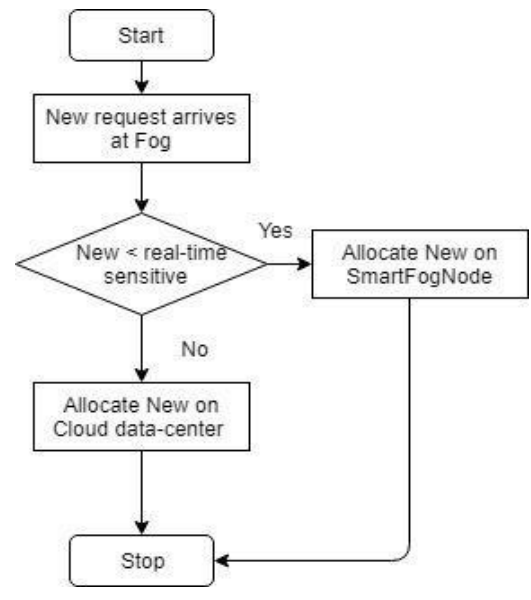

Figure 2: Flow chart of request allocation

\section{Cloud Layer}

This layer consists of cloud servers and data-centers that provide processors and storage to the application's computing at the cloud layer. The less-time sensitive data and processed data coming from the fog layer are processed and stored, respectively. The processed data put less overhead on the cloud servers and data-centers. 


\section{THE ENERGY ASPECT OFFATEH}

In the real implementation of the proposed architecture, reducing the energy consumption of IoT devices is a

challenging task. While implementing multi-hop configuration at large-scale, the data delivery becomes degrade due to the network congestion that results in increasing packet drop ratio, especially with those which are far away from the Smart- Fog node. Moreover, this increased data traffic causes the depletion of batteries drastically. Subsequently, the overall data captured by sensors gets disrupted.

The IoT-based devices are to be more energy-efficient and more cost-effective because they are intended to run for months or years. In our proposed framework, these devices do not make communication with the SmartFog nodes or cloud data-centers directly. Here, an AgentNode is used as a border router, which acts as a single point of contact at the physical layer. The agent node provides an interface and aggregates the information received from the various sensor nodes. The energy consumed by the AgentNode is not taken into account because it is connected to power and does not collect its data.

In the proposed framework, choosing the appropriate routing protocols is a big challenge. RPL supports the huge addressing space, which is required for a large number of IoT objects. The RPL provides solutions to the devices that are running at large scale networks using low power and lowcost communications. As RPL is a tree-oriented protocol, a DODAG is build corresponding to the network construction stage. It builds downward and upward paths between the layers by using the neighbor discovery process.

Further, for making network stable (less convergence time and less consumption of scarce resources), Objective Function (OF) is designed. It is the essential aspect of the RPL protocol, which selects the path to the destination based on the hop count. The objective function OF0 is less efficient while dealing with low power lossy networks. So a novel Objective Function is designed, which is known as ETX (Expected Transmission Count). The ETX is calculated as :

ETXLink $=1 /$ (LQForward $*$ LQReverse) $(1)$

Here, ETX Link is the expected transmission count, LQForward is the link quality in forwarding direction, and LQReverse is the link quality in reverse direction between two nodes.

Hop count and Expected Transmission Count (ETX) are the two routing metrics for implementing RPL in the ContikiHop Count metric which shows the total number of hops utilized whereas ETX represents the expected number of transmissions required to send a packet over the link. To evaluate those routing metrics, four standard performance metrics are used Network convergence, Energy consumption, Packet Latency, and Packet Delivery Ratio (PDR).

Packet Latency: It is the time consumed by a packet moving from the sender to the sink. Therefore, packet latency is the sum of the average of the latencies of nodes all the packages in the network. Packet Delivery Ratio (PDR) is computed as the ratio of the total number of packets received successfully at the sink to total the number of sent packets to sink.

\section{EXPERIMENTAL SETUP}

To perform the experimental setup for the proposed framework, the simulation is divided into two folds. Firstly, the construction of a real IoT environment as a testbed for evaluating the proposed three-layer architecture using IoT devices is very costly and not suitable for conducting repeatable experiments in a controlled environment. Therefore, an open-source Java-based simulator called ifogSim and Contiki Cooja is used for the simulations. The ifogSim enables the modeling and simulation of the fogcomputing environment. It efficiently affects the IoT devices for computing delays in different scenarios as well as energy consumption of the data-centers located at cloud. For transforming the environment, we design a three-level structure, as shown in Figure 5. At level 0 cloud data-centers are placed, and in the next level 1 , fog devices are places as

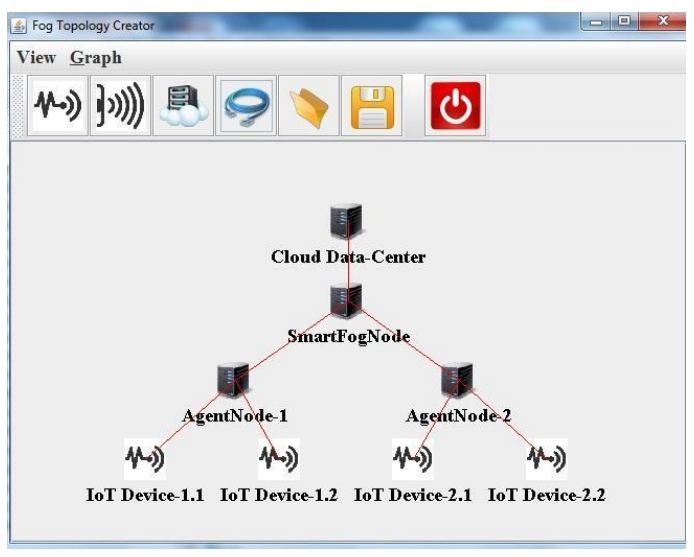

Figure 5: Topology Simulation for FATEH

SmartFogNode and at the last level, 2 AgentNode and IoT devices The following hierarchical manner. The latency configuration between various components of our proposed framework is considered. To obtain correctness of the result during the experiments, multiple topologies are used with different placement strategies. The configuration of each physical entity such as cloud data-centers, gateways, etc. is considered as listed. The processing capability of each device is expressed in millions of instructions per second (MIPS), whereas bandwidth is measured in the form of Up-link and Down-link. The experiment is performed in a repeatable manner to collect the result. The energy consumption of cloud data-centers is calculated for various strategies. Further, the delay corresponds to each layer is computed to find the total delay in our framework. The latency of all individual devices is also calculated in both situations, i.e. when all devices directly interact with the low-cost cloud and with the presence of fog layer.

On the other hand, for evaluation of the energy aspect of FATEH, an operating system called Contiki, along with its simulator COOJA are used to implement RPL protocol. The operating system used in the proposed system for simulating energy consumption is open-source which specifically used for 
multi-tasking in wireless sensor networks (WSN). Further, the COOJA simulator is used to simulate sensor networks running on the Contiki operating system. The experimented is simulated for analyzing the RPL's performance in WSN.

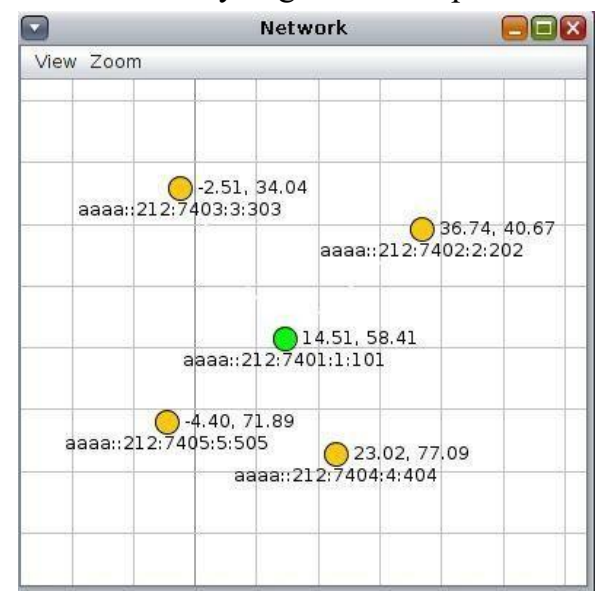

Fig.7 Node creation

The creation of nodes is represented in Figure7.

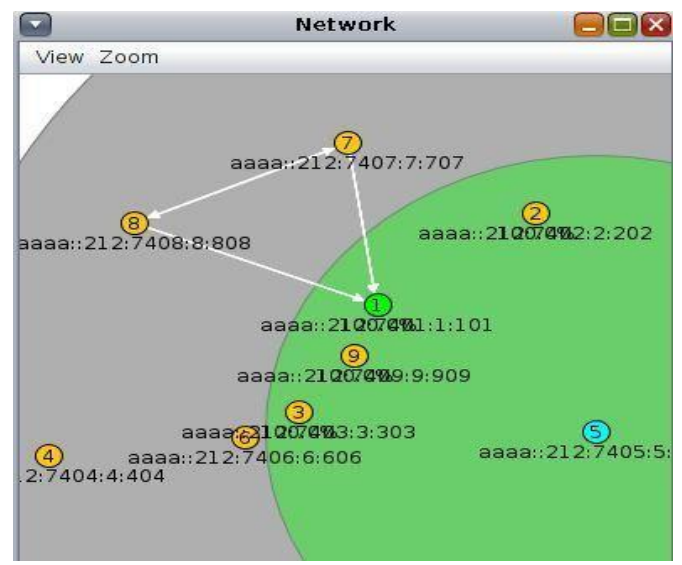

The configuration setup for the COOJA simulator is shown. DODAG (destination-oriented directed acyclic graph) creation is shown in figure 8 , which is used for implementing RPL protocol.

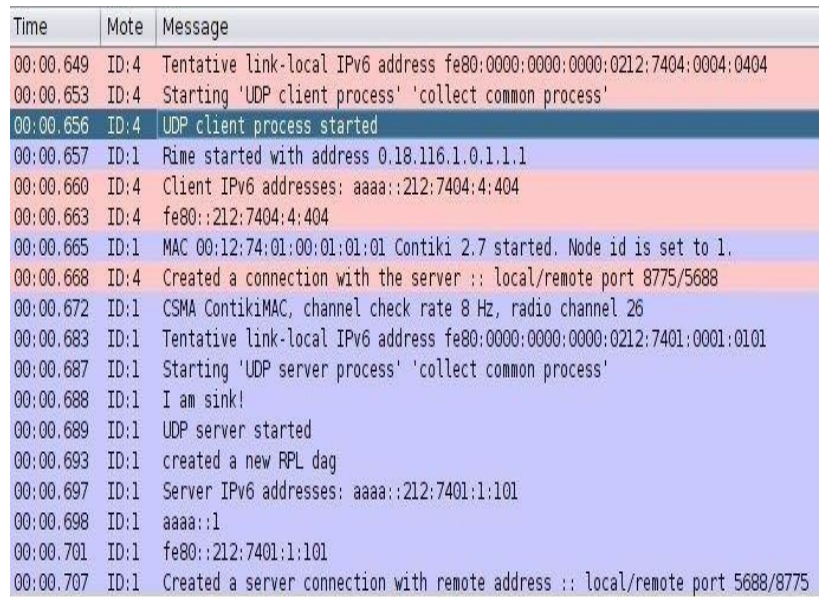

Fig 8. DODAG Creation

\section{RESUlts \& DiscUSSION}

The simulation results are compared and analyzed. Figure 10 shows the energy consumption of the system in both conditions using traditional configuration and using FATEH configuration. It clearly shows when the fog layer is not in use, the energy consumption of cloud data-centers becomes very high. Whereas by using the fog layer in the proposed framework, the total energy consumed became low.

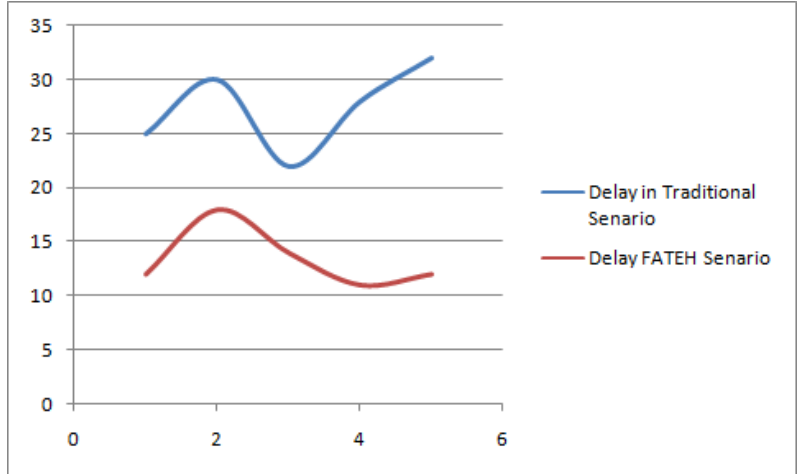

Fig 9. Delay count Comparision

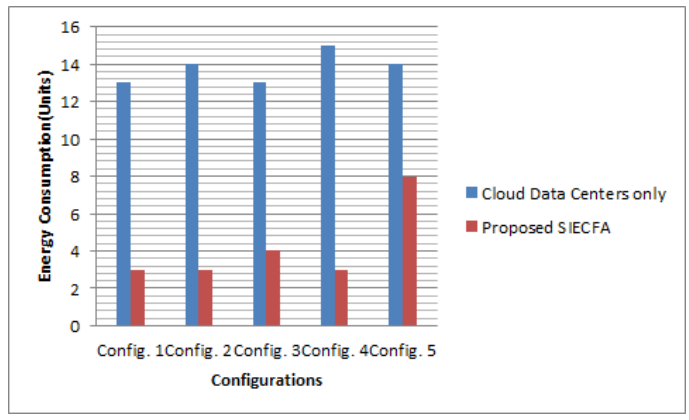

Figure 10. Comparison of Energy Consumption

Figure 9 depicts that the delay found in the FATEH scenario, which is calculated as the sum of delay between Agent Nodes and SmartFog nodes and SmartFogNodes and the cloud datacenters is less than the total delay. It is noted here that at the FATEH scenario, all computations for the data are done at SmartFogNodes only In the second fold of experiment, the performance evaluation of all IoT devices utilized under the RPL protocol is shown in Figure 6. It represents performance evaluation of RPL protocol at various stated metrics of CPU (when CPU is in active mode). The network convergence is shown in Figure 6

(a). Figure 6 (b) depicts the total power consumption. Subsequently, no power is consumed while the CPU is in deactivate mode. Also, the power consumed during LPM (Low power mode) Radio Listen and Radio Transmit respectively are shown. The packet latency is shown by the graph. Figure 6 (c). The graph is shown in Figure 6 (d) depicts the packet delivery ratio. Finally, Figure 6 (e) represents the expected transmission count. 


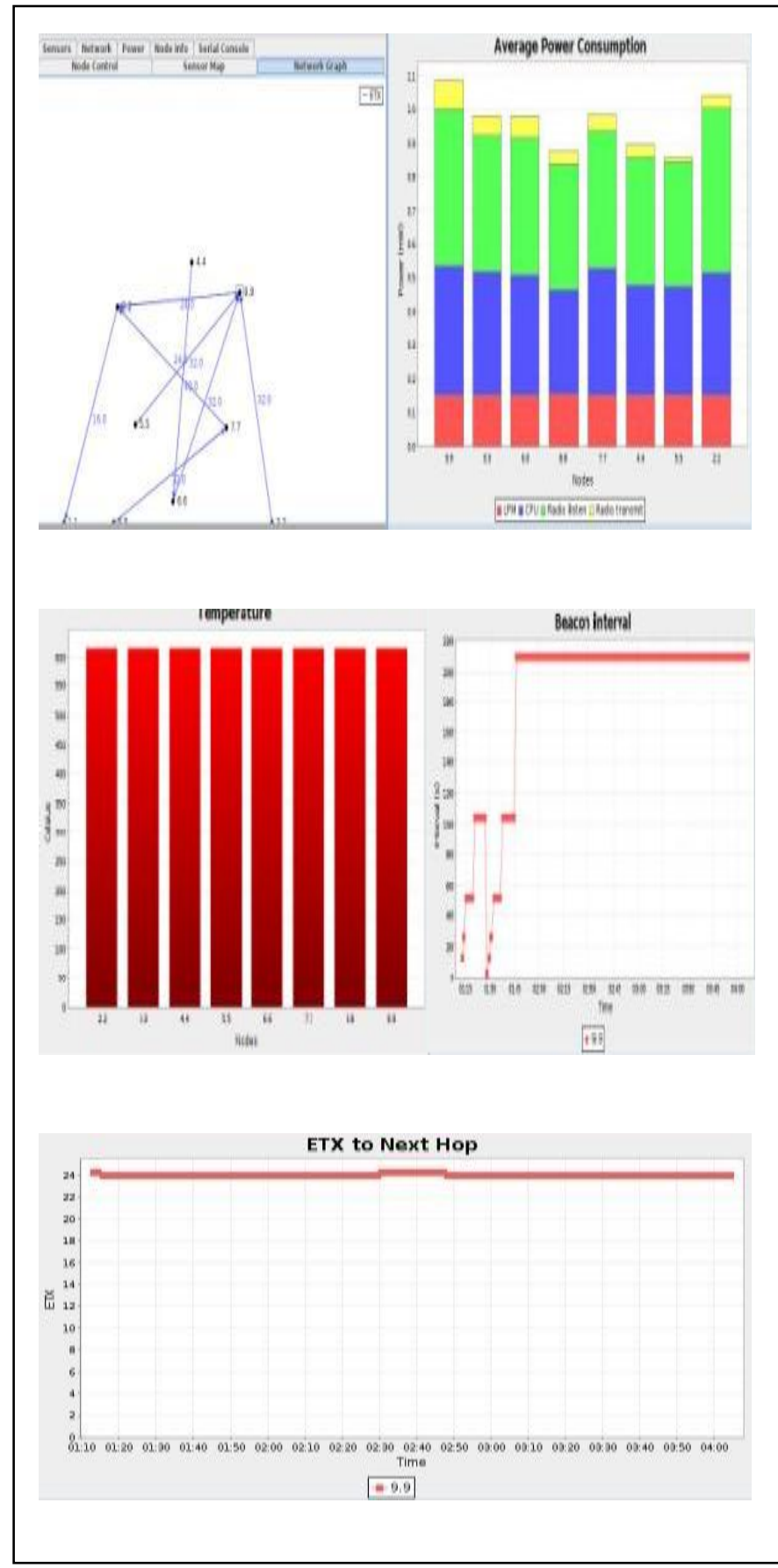

Fig 6. Performance Evaluation of RPL

\section{CONCLUSION}

The FATEH facilitates Quality of Service framework using fog computing for smart city applications. It makes an IoT based system more efficient to achieve better future results and decision making. The design of FATEH is presented with three layers, where the real-time monitoring of the data is collected through AgentNode and processed at the proposed SDN based SmartFogNode. The fog manager efficiently categorizes the data into real-time sensitive to be processed at the fog layer and less-time sensitive data to be processed at cloud layer. To achieve the QoS RPL is utilized for optimum use of energy in all nodes.

\section{REFERENCES}

[1] Baccarelli, E., Cordeschi, N., Mei, A., Panella, M., Shojafar, M., and Stefa, J. Energy efficient dynamic traffic offloading and reconfiguration of networked data centers for big data stream mobile computing: review, challenges, and a case study. IEEE Network 30, 2 (2016), 54-61.

[2] Bonomi, F., Milito, R., Natarajan, P., and Zhu, J. Fog computing: A platform for internet of things and analytics. In Big data and internet of things: A roadmap for smart environments. Springer, 2014, pp. 169-186.

[3] Brewster, C., Roussaki, I., Kalatzis, N., Doolin, K., and Ellis, K. Iot in agriculture: Designing a europe-wide large-scale pilot. IEEE Communications Magazine 55, 9 (2017), 26-33.

[4] Chang, C. K., Reisman, S., and Tovar, E. Advances in learning technologies. Computer 50, 5 (2017), 14-17.

[5] Deng, R., Lu, R., Lai, C., Luan, T. H., and Liang, H. Optimal workload allocation in fogcloud computing toward balanced delay and power consumption. IEEE Internet of Things Journal 3, 6 (2016), 1171-1181.

[6] dos Santos, P. M. S. Wireless protocols and channel estimation for data gathering with mobile nodes.

[7] Gubbi, J., Buyya, R., Marusic, S., and Palaniswami, M. Internet of things (iot): A vision, architectural elements, and future directions. Future generation computer systems 29, 7 (2013), 1645- 1660.

[8] Jalali, F., Hinton, K., Ayre, R., Alpcan, T., and Tucker,

R. S. Fog computing may help to save energy in cloud computing. IEEE Journal on Selected Areas in Communications 34, 5 (2016), 1728-1739.

[9] Li, J., Jin, J., Yuan, D., and Zhang, H. Virtual fog: A virtualization enabled fog computing framework for internet of things. IEEE Internet of Things Journal 5, 1 (2018), 121-131.

[10] Liu, Y., Fieldsend, J. E., and Min, G. A framework of fog computing: Architecture, challenges and optimization. IEEE Access (2017).

[11] Ning, H., and $\mathrm{Hu}, \mathrm{S}$. Technology classification, industry, and education for future internet of things. International Journal of Communication Systems 25, 9 (2012), 1230-1241.

[12] Peng, M., Wang, Y., Dang, T., and Yan, Z. Cost-efficient resource allocation in cloud radio access networks with heterogeneous fronthaul expenditures. IEEE Transactions on Wireless Communications (2017).

[13] Premsankar, G., Di Francesco, M., and Taleb, T. Edge computing for the internet of things: A case study. IEEE Internet of Things Journal 5, 2 (2018), 1275-1284.

[14] Vaquero, L. M., and Rodero-Merino, L. Finding your way in the fog: Towards a comprehensive definition of fog computing. ACM SIGCOMM Computer Communication Review 44, 5 (2014), 27-32.

[15] Wu, T., Wu, F., Redoute, J.-M., and Yuce, M. R. ' An autonomous wireless body area network implementation towards iot connected healthcare applications. IEEE Access 5 (2017), 11413-11422.

[16] Yang, S. Iot stream processing and analytics in the fog. arXiv preprint arXiv:1705.05988 (2017).

[17] Yu, L., Jiang, T., and Zou, Y. Fog-assisted operational cost reduction for cloud data centers. IEEE Access 5 (2017), 13578-13586. 
Design and develop Quality of Service framework using fog computing for smart city applications

\section{AUTHORS PROFILE}

Prabhdeep Singh is doing Ph.D. from Punjabi University and has completed his M.E. from Thapar University,Patiala. $\mathrm{He}$ has published more than 20 papers in International Journals and Conferences. His areas of research are smart city development, IoT, Cloud computing, Fog computing, Big Data, and Edge computing. He is a lifetime member of broadcast engineering society.

Dr. Rajbir Kaur has completed Ph.D. in Signa System. She has published more than 25 papers in International Journals and Conferences. Her areas of research are Signal System, Cloud computing, Fog computing, and Edge computing. 